\title{
Screening of Amazonian plants from the Adolpho Ducke forest reserve, Manaus, state of Amazonas, Brazil, for antimicrobial activity
}

\author{
Ana Lúcia Basílio Carneiro, Maria Francisca Simas Teixeiraㅁ, Viviana Maria Araújo de Oliveira², \\ Ormezinda Celeste Cristo Fernandes ${ }^{3}$, Gláucia Socorro de Barros Cauper ${ }^{4}$, \\ Adrian Martin Pohlit ${ }^{4}+$
}

\begin{abstract}
Departamento de Morfologia 'Laboratório de Micologia, Instituto de Ciências Biológicas, Universidade Federal do Amazonas, Manaus, AM, Brasil ${ }^{2}$ Fundação de Hematologia e Hemoterapia do Amazonas-HEMOAM, Manaus, AM, Brasil ${ }^{3}$ Centro de Pesquisa Leônidas e Maria Deane-Fiocruz, Manaus, AM, Brasil ${ }^{4}$ Laboratório de Princípios Ativos da Amazônia-LAPAAM, Coordenação de Pesquisa em Produtos Naturais-CPPN, Instituto Nacional de Pesquisa da Amazônia-INPA, Av. André Araújo 2936, 69011-970 Manaus, AM, Brasil
\end{abstract}

Tropical forests are species-rich reserves for the discovery and development of antimicrobial drugs. The aim of this work is to investigate the in vitro antimicrobial potential of Amazon plants found within the National Institute on Amazon Research's Adolpho Ducke forest reserve, located in Manaus, state of Amazonas, Brazil. 75 methanol, chloroform and water extracts representing 12 plant species were tested for antimicrobial activity towards strains of Mycobacterium smegmatis, Escherichia coli, Streptococcus sanguis, Streptococcus oralis, Staphylococcus aureus and Candida albicans using the gel-diffusion method. Active extracts were further evaluated to establish minimum inhibitory concentrations (MIC) and antimicrobial profiles using bioautography on normal-phase thin-layer chromatography plates. Diclinanona calycina presented extracts with good antimicrobial activity and S. oralis and M. smegmatis were the most sensitive bacteria. D. calycina and Lacmellea gracilis presented extracts with the lowest MIC $(48.8 \mu \mathrm{g} / \mathrm{ml})$. D. calycina methanol and chloroform leaf extracts presented the best overall antimicrobial activity. All test organisms were sensitive to $\mathrm{D}$. calycina branch chloroform extract in the bioautography assay. This is the first evaluation of the biological activity of these plant species and significant in vitro antimicrobial activity was detected in extracts and components from two species, $\mathrm{D}$. calycina and $\mathrm{L}$. gracilis.

Key words: plant extract - antibacterial - antifungal - Diclinanona calycina - Lacmellea gracilis - bioautography

The Amazon region is the largest tropical forest in the world. It occupies almost half the South American continent and is a center of biological diversity. Aside from its importance in the global ecological equilibrium and as a location of increasing ecological tourism, this region is a very rich source of species for agriculture, plant domestication and medicinal applications. Approximately 125,000 plant species are in tropical forests which continue to be a great reservatory for the discovery of new bioactive molecules and phytotherapeutic agents. However, the pharmacological potential of only about $1 \%$ of all tropical plant species has been evaluated. Also, Brazil has catalogued only about $0.4 \%$ of its flora. It is estimated that ca. $60 \%$ of all commercially available or clinical-phase antitumor and antimicrobial drugs are of natural origin (Montanari \& Bolzani 2001, Gurib-Fakim 2006, Turolla \& Nascimento 2006).

Despite the existence of publications on the traditional (Fenner et al. 2006, Giorgetti et al. 2007) and pharmaceutical (Moreira et al. 2006) uses of plant species found

Finnancial support: PPG-7/CNPq (557106/2005-2), PPBio/MCT/INPA + Corresponding author: ampohlit@inpa.gov.br

Received 15 May 2007

Accepted 12 February 2008 in Brazil there are still relatively few scientific studies on the pharmacological and toxicological properties of plants from Brazil. There are also very few examples of the discovery of bioactive substances from Brazilian biodiversity which have served as prototypes for development of phytotherapeutic agents and pharmaceuticals in Brazil. Furthermore, many active substances present in native plant extracts from Brazil have still not been identified so this is an area which needs to be explored (Moreira et al. 2006). As examples of Brazilian plant-derived commercial products one should mention Ierobina ${ }^{\circledR}$, which is used in the treatment of indigestion (Botion et al. 2005), and the anti-inflammatory phytotherapeutic agent Acheflan ${ }^{\circledR}$ (Giorgetti et al. 2007). The relatively scarce interaction between mainly public universities and companies in Brazil as well as Brazilian patent law have been drawbacks for transformation of research results into developed products and patents (Moreira et al. 2006, Giorgetti et al. 2007).

Native Amazon forest plants have been poorly studied and there are no good estimates of the number of species which have been studied or which have application to human health. The use of flora from this region has been modest in relation to its strategic value in the development of local products. Our group has been actively screening medicinal and other plants found in the Brazilian Amazon region for larvicidal and insecticidal (Pohlit et al. 2004) as well as cytotoxic (Quignard et al. 2003, Quignard et al. 2004) properties, among other biological activities. 
Many plant species have demonstrated antibacterial (Koo et al. 2000, Melo et al. 2006) and antifungal (Motsei et al. 2003, Gayoso et al. 2004) properties. However, little research has focused on the evaluation of plant species for activity against microorganisms from the oral cavity (Pereira et al. 2005, Bandeira et al. 2006). This is especially true for plants from the Amazon region.

Therefore, there is a general lack of scientific investigation which can provide more realistic knowledge of the potential of Amazon biodiversity. As a means of contributing to knowledge of the pharmacological potential of the Amazon flora, the present work evaluated 12 Amazonian plant species - having no known previous pharmacological study- for in vitro antimicrobial activity in bacteria and a fungus species associated with the human oral cavity diseases, as well as other diseases.

\section{MATERIALS AND METHODS}

Plant selection - Species identified by the Flora Project (Ribeiro et al. 1999) in the National Institute on Amazon Research's Adolpho Ducke forest reserve in Manaus, state of Amazonas, Brazil were initially contemplated for study. These plant specimens had been previously catalogued so that their botanic identities (based on voucher samples at the Instituto Nacional de Pesquisa da Amazônia-INPA Herbarium) and exact locations in the Ducke reserve were readily available. After a literature search, several hundred plant species for which no ethnobotanic, phytochemical, pharmacological or medicinal information were available were identified. From this larger group of plants, the 12 plant species were chosen for the purposes of this study (Table I) based on: (1) the ease of access and availability of sufficient plant materials in the Ducke reserve; and (2): their broad representative value of the local, unexplored flora (the species under study belong to 11 distinct botanic families).

Plant collection - Plants were collected between January and June 2004 in the Ducke reserve. Plant specimens were located in the field based on the Ducke reserve trail map (Flora Project 2006) and geographical coordinates were obtained from the Flora Project database. Plant materials (leaves, branches, vine or bark) were initially dried in an air-conditioned, dehumidified room, then further dried in an oven at ca. $40^{\circ} \mathrm{C}$ for a total of ca. seven days, and then finally ground.

Preparation of extracts - Plant methanol and chloroform extracts were prepared by continuous extraction of ground plant material in a soxhlet apparatus for $18 \mathrm{~h} \mathrm{(3}$ $\mathrm{x} 6 \mathrm{~h}$ ) followed by rotary evaporation and freeze-drying. Water extracts were prepared by infusion followed by filtration and total evaporation of the filtrate. Extracts were stored in a freezer at $-20^{\circ} \mathrm{C}$.

Microorganisms used - Standardized strains from the American type culture collection (ATCC) and the Department of Antibiotics, Federal University of Pernambuco (DAUPE) were used in bioassays. The Grampositive bacteria were Mycobacterium smegmatis (ATCC 607), Streptococcus oralis (ATCC 10557), Streptococcus sanguis (ATCC 15300) and Staphylococcus aureus (DAUPE). The Gram-negative bacterium was Escherichia coli (DAUPE 224). Antifungal activity was evaluated using a clinical strain of Candida albicans from the collection of the Department of Parasitology, Federal University of Amazonas. Organisms were maintained at $4^{\circ} \mathrm{C}$ on brain heart agar (bacteria) and sabouraud (SAB) (C. albicans). For the antibacterial tests, organisms were grown overnight in brain heart infusion followed by incubation at $37^{\circ} \mathrm{C}$. Before the test, C. albicans was cultured on $\mathrm{SAB}$ at $37^{\circ} \mathrm{C}$ for $48 \mathrm{~h}$.

Antimicrobial susceptibility testing - Evaluation of the antimicrobial activity of plant extracts was carried out in Petri dishes using the agar diffusion method (Alves et al. 2000, Chah et al. 2006, Melo et al. 2006) by perforating the culture medium and charging each cavity with extract dissolved in dimethylsulphoxide (DMSO), ethanol or sterilized distilled water at a concentration of $50 \mathrm{mg} / \mathrm{ml}$.

For antibacterial tests, $25 \mathrm{ml}$ of Mueller Hinton (MH) culture medium was used. For antifungal tests, SAB was used (C. albicans). Microbial suspensions were prepared

TABLE I

Families, species names and other information for the plants from the Ducke Reserve under study

\begin{tabular}{|c|c|c|c|}
\hline Family & Species & Synonym & Common Name \\
\hline Anacardiaceae & Thyrsodium spruceanum Benth. & Thyrsodium paraense Huber & Breu-de-leite ${ }^{a}$ \\
\hline Annonaceae & Diclinanona calycina Benoiste & Xylopia calycina Diels & Envireira $^{a}$ \\
\hline Apocynaceae & Lacmellea gracilis (Mull.Arg.) Markgr. & Zschokkea gracilis Mull & $\begin{array}{l}\text { Caramurizinho, } \\
\text { sorvinha }^{b}\end{array}$ \\
\hline Icacinaceae & Pleurisanthes parviflora (Ducke) Howard & - & - \\
\hline Olacaceae & Chaunochiton kappleri (Sagot ex Engl.) Ducke & Chaunochiton breviflorum Ducke & - \\
\hline Passifloraceae & Dilkea johannesii Barb. Rodr. & Dilkea ulei Harms & $\begin{array}{l}\text { Grão de bode }{ }^{b}, \\
\text { Pacarupia }^{c}\end{array}$ \\
\hline Polygalaceae & Moutabea guianensis Aubl. & - & - \\
\hline Rhizophoraceae & Sterigmapetalum obovatum Kuhlm. & - & - \\
\hline Sapotaceae & Elaeoluma nuda (Baehni) Aubrév. & Pouteria nuda & - \\
\hline Sapotaceae & Sarcaulus brasiliensis (A. Dc.) Eyma & Sarcaulus macrophyllus (Mart) Radlk & Guajara $^{a}$ \\
\hline Thymelaeaceae & Schoenobiblus daphnoides Mart. & - & - \\
\hline Violaceae & Paypayrola grandiflora Tul. & Paypayrola ventricosa Tul. & - \\
\hline
\end{tabular}

References for species names: $a$ : Ribeiro et al. (1999); $b$ : Silva et al. (1977); $c$ : Corrêa (1978). 
and the density was adjusted in the tube to 1 on the McFarland scale. After solidification, medium was inoculated with microbial suspension $(100 \mu \mathrm{l})$ with the aid of a swab. After $10 \mathrm{~min}$, the agar was perforated so as to yield five circular, equidistant cavities (diameter $6 \mathrm{~mm}$ each). An aliquot of extract $(50 \mu \mathrm{l}$ of a $50 \mathrm{mg} / \mathrm{ml}$ solution) and positive (rifampicin and nistatin) and negative (solvent blank) controls were transferred to cavities. After the incubation period ( 24 and $48 \mathrm{~h}$ ) at $37^{\circ} \mathrm{C}$, plates were examined and extract activity was evaluated by measurement of inhibition zone diameters (in mm). Tests were performed in triplicate and average halo diameters were determined. Rifampicin ( $1 \mathrm{mg} / \mathrm{ml})$ was used as positive control for bacteria and nistatin $(1 \mathrm{mg} / \mathrm{ml})$ was used for tests involving C. albicans. The negative controls were DMSO, ethanol and sterilized, distilled water in accordance with the solvents used to dissolve each extract. A negative control containing culture medium was also used.

Determination of minimum inhibitory concentrations (MIC) - Extracts considered very active in the above susceptibility tests (inhibition halo $>20 \mathrm{~mm}$ in the agar diffusion test) were next evaluated to determine MIC. This was carried out by dilution in solid culture medium by adapting techniques proposed by Melo et al. (2006). For the test, $35 \mathrm{ml}$ of agar were prepared. $\mathrm{MH}$ was used for bacteria and $\mathrm{SAB}$ for C. albicans, using homogenization with $1.5 \mathrm{ml}$ of the microorganism suspension having a density equal to 1 on the McFarland scale. Thirty $4 \mathrm{~mm}$ diameter orifices were prepared and inoculated, in triplicate, with $15 \mu \mathrm{l}$ of each of a series of 10 dilutions of each crude extract stock solution $(50 \mathrm{mg} / \mathrm{ml})$. MICs correspond to the lowest concentration which inhibited the growth of the microorganism.

Thin-layer chomatography (TLC) - Aluminum-backed commercial TLC silica gel GF $_{254}$ plates (MERCK) were used. The extracts were applied to plates and eluted with chloroform/acetone (90:10). Eluted TLC plates were observed under ambient lighting and illuminated with an ultraviolet lamp at 254 and $366 \mathrm{~nm}$.

Bioautography - Active extracts were evaluated through the bioautography technique adapted from Alves et al. (2001) and Holetz et al. (2002). Briefly, microorganism culture (1 on McFarland's scale, $500 \mu \mathrm{l})$ and developing agent (2,3,5-triphenyltetrazolium chloride, $1 \mathrm{ml})$ in aqueous solution (this reagent aids in the observation of inhibition zones) were added to the culture medium $(25 \mathrm{ml})$ at $50^{\circ} \mathrm{C}$. After homogenization, this mixture was poured over eluted chromatograms and these were placed in Petri dishes and incubated at $37^{\circ} \mathrm{C}$. Plates were observed after incubation for 24 and $48 \mathrm{~h}$. Inhibition was evidenced by clear zones on the plates where no ostensible microorganism growth had occurred.

\section{RESULTS}

In the screening for antimicrobial activity, after a period of $24 \mathrm{~h}$, the largest average inhibition halos resulted from the action of the methanol and chloroform extracts of Diclinanona calycina. S. oralis e M. smegmatis were the most sensitive organisms in this test. Of the 75 extracts evaluated for inhibitory effects on $M$. smegmatis, only ten presented negative results (no inhibition halos), while 11 allowed for the formation of inhibition halos with diameters greater than $20 \mathrm{~mm}$. Extracts of Pinus parviflora presented slight or no activity towards the microorganisms used in testing. These and other results are presented in Table II. Antimicrobial substances used as positive controls presented inhibition halos as expected, differing from negative controls (DMSO, water, ethanol blanks), which in general did not present inhibition halos.

This preliminary screening of all extracts permitted triage of promising antimicrobial extracts (presenting inhibition halo diameter $\geq 20 \mathrm{~mm}$ ). 34 halos with diameters $\geq 20 \mathrm{~mm}$, corresponding to 23 different extracts, were observed. Active extracts were further tested in dilution for the determination of minimum inhibition concentrations (MIC) by diffusion in solid culture medium. $D$. calycina e Lacmella gracilis presented the lowest MIC values $(48.8 \mu \mathrm{g} / \mathrm{ml})$. D. calycina leaf methanol and chloroform extracts presented the best inhibitory effects on E. coli, S. oralis, S. aureus and S. sanguis. L. gracilis branch chloroform extract presented the lowest MIC on M. smegmatis $(48.8 \mu \mathrm{g} / \mathrm{ml})$. Under the experimental conditions used, none of the eight tested extracts inhibited $C$. albicans growth (Table III).

Results of bioautography screening revealed several (mainly chloroform) extracts which exhibited inhibition zones corresponding to substances of differing polarities/ retention factors $\left(\mathrm{R}_{\mathrm{fs}}\right)($ Table IV). D. calycina extracts presented the best bioautography results. All microorganisms tested in this study were sensitive to $D$. calycina branch chloroform extract and most of these microorganisms (M. smegmatis, S. oralis, S. aureus and S. sanguis) showed sensitivity to more than one chemical component of this extract. In this way, screening and MIC determinations by diffusion in agar and bioautography permitted identification of active extracts containing antibacterial components. In this way, it was observed that in screening of extracts for antimicrobial activity it can be very important to use a variety of test methods which can, as in the present case, reveal information about the most active extracts and also reveal information on the number of bioactive components.

\section{DISCUSSION}

Microorganisms selected for this study are important human oral cavity pathogens or are of interest because they represent diseases which are of interest to public health. S. sanguis, for example, is one of the predominant colonizers of teeth. It has been isolated from human dental plaque, tongue, saliva, root canals, periapical and periodontal infections and it causes endocarditis. Cavities produced by $S$. sanguis occur principally in tooth fissures (Hamada \& Slade 1980, Coykendall 1989, Piovano 1999, Wade et al. 2005).

E. coli is recommended as control group in antimicrobial susceptibility tests for enterobacteria. Also, E. coli and $S$. aureus are included among the most common agents of hospital infections and are detected in cases of meningitis and are potential respiratory pathogens (Gendron et al. 2000, Melo-Souza, 2000, Esmerino et al. 2005). 
TABLE II

Average inhibition zone diameters (in $\mathrm{mm}$ ) observed for plant extracts (well concentrations $50 \mathrm{mg} / \mathrm{ml}$ ) in the agar diffusion method for the different test microorganisms used

\begin{tabular}{|c|c|c|c|c|c|c|c|c|}
\hline \multirow[b]{2}{*}{ Species } & \multirow[b]{2}{*}{ Part } & \multirow[b]{2}{*}{ Solvent } & \multicolumn{6}{|c|}{ Average inhibition zone diameter (mm) } \\
\hline & & & Ms & $\mathrm{Ec}$ & $\mathrm{Sa}$ & So & Ss & $\mathrm{Ca}$ \\
\hline \multirow[t]{6}{*}{ C. kappleri } & \multirow[t]{3}{*}{ leaf } & $\mathrm{CHCl}_{3}$ & 19 & 10 & 10 & 9 & 10 & - \\
\hline & & $\mathrm{CH}_{3} \mathrm{OH}$ & 15 & 14 & 12 & 15 & 13 & 10 \\
\hline & & $\mathrm{H}_{2} \mathrm{O}$ & 14 & 16 & 14 & 15 & 14 & 11 \\
\hline & \multirow[t]{3}{*}{ branch } & $\mathrm{CHCl}_{3}$ & 14 & - & 11 & 12 & 16 & 12 \\
\hline & & $\mathrm{CH}_{3} \mathrm{OH}$ & 15 & 15 & 9 & 15 & 15 & 15 \\
\hline & & $\mathrm{H}_{2} \mathrm{O}^{3}$ & 15 & - & 14 & 16 & 13 & 12 \\
\hline \multirow[t]{6}{*}{ D. calycina } & \multirow[t]{3}{*}{ leaf } & $\mathrm{CHCl}_{3}$ & 26 & 20 & 20 & 23 & 22 & 15 \\
\hline & & $\mathrm{CH}_{3} \mathrm{OH}$ & 40 & 20 & 17 & 18 & 20 & - \\
\hline & & $\mathrm{H}_{2} \mathrm{O}$ & 16 & 16 & - & 15 & 14 & 15 \\
\hline & \multirow[t]{3}{*}{ branch } & $\mathrm{CHCl}_{3}$ & 15 & 15 & 14 & 15 & 18 & 10 \\
\hline & & $\mathrm{CH}_{3} \mathrm{OH}$ & 21 & _- & 11 & 13 & 16 & 9 \\
\hline & & $\mathrm{H}_{2} \mathrm{O}$ & 15 & 11 & - & 14 & 9 & 13 \\
\hline \multirow[t]{6}{*}{ D. johannesii } & \multirow[t]{3}{*}{ leaf } & $\mathrm{CHCl}_{3}$ & 12 & 17 & - & 8 & 9 & - \\
\hline & & $\mathrm{CH}_{3} \mathrm{OH}$ & 14 & 11 & 8 & - & - & - \\
\hline & & $\mathrm{H}_{2} \mathrm{O}$ & 8 & 12 & 8 & 9 & - & - \\
\hline & \multirow[t]{3}{*}{ branch } & $\mathrm{CHCl}_{3}$ & 11 & - & 13 & 11 & 15 & - \\
\hline & & $\mathrm{CH}_{3} \mathrm{OH}$ & 15 & 11 & - & 10 & 10 & - \\
\hline & & $\mathrm{H}_{2} \mathrm{O}$ & - & 20 & 9 & 10 & 9 & - \\
\hline \multirow[t]{9}{*}{ E. nuda } & \multirow[t]{3}{*}{ leaf } & $\mathrm{CHCl}_{3}$ & 12 & 10 & - & 8 & 9 & - \\
\hline & & $\mathrm{CH}_{3} \mathrm{OH}$ & 10 & 11 & - & 14 & 12 & 20 \\
\hline & & $\mathrm{H}_{2} \mathrm{O}$ & 16 & 20 & 10 & 16 & 12 & 15 \\
\hline & \multirow[t]{3}{*}{ branch } & $\mathrm{CHCl}_{3}^{2}$ & 17 & 15 & 10 & 20 & 10 & - \\
\hline & & $\mathrm{CH}_{3} \mathrm{OH}$ & 15 & - & 11 & 14 & 13 & - \\
\hline & & $\mathrm{H}_{2} \mathrm{O}$ & 15 & 9 & 8 & 11 & 10 & 10 \\
\hline & bark & $\mathrm{CHCl}_{3}$ & 15 & 10 & 10 & 10 & 10 & - \\
\hline & & $\mathrm{CH}_{3} \mathrm{OH}$ & 10 & 12 & 10 & 13 & 13 & - \\
\hline & & $\mathrm{H}_{2} \mathrm{O}$ & 15 & 16 & - & 12 & 8 & 12 \\
\hline L. gracilis & leaf & $\mathrm{CHCl}_{3}$ & - & 15 & - & 25 & 10 & 10 \\
\hline & & $\mathrm{CH}_{3} \mathrm{OH}$ & 12 & 10 & - & - & - & 15 \\
\hline & & $\mathrm{H}_{2} \mathrm{O}$ & 12 & 12 & - & - & - & - \\
\hline & branch & $\mathrm{CHCl}_{3}$ & 21 & 18 & 15 & 24 & 15 & 12 \\
\hline & & $\mathrm{CH}_{3} \mathrm{OH}$ & 10 & 12 & 10 & 11 & 11 & - \\
\hline & & $\mathrm{H}_{2} \mathrm{O}$ & 11 & - & 9 & 11 & - & 23 \\
\hline M. guianensis & leaf & $\mathrm{CHCl}_{3}$ & 12 & 11 & 11 & 10 & 9 & 10 \\
\hline & & $\mathrm{CH}_{3} \mathrm{OH}$ & 10 & 10 & 8 & 10 & 8 & - \\
\hline & & $\mathrm{H}_{2} \mathrm{O}$ & - & - & 21 & - & 8 & - \\
\hline & branch & $\mathrm{CHCl}_{3}$ & 12 & 10 & 12 & 12 & 15 & 8 \\
\hline & & $\mathrm{CH}_{3} \mathrm{OH}$ & 19 & 10 & 15 & 19 & 10 & 8 \\
\hline & & $\mathrm{H}_{2} \mathrm{O}$ & - & 12 & - & - & 10 & 20 \\
\hline P. grandiflora & leaf & $\mathrm{CHCl}_{3}$ & - & 11 & 11 & 9 & 9 & 10 \\
\hline & & $\mathrm{CH}_{3} \mathrm{OH}$ & 12 & 14 & 14 & 13 & 14 & 12 \\
\hline & & $\mathrm{H}_{2} \mathrm{O}$ & - & 12 & 13 & 12 & 14 & 12 \\
\hline & branch & $\mathrm{CHCl}_{3}$ & 12 & 12 & - & 14 & 14 & 10 \\
\hline & & $\mathrm{CH}_{3} \mathrm{OH}$ & 25 & 12 & 13 & 15 & 9 & 15 \\
\hline & & $\mathrm{H}_{2} \mathrm{O}$ & 10 & - & 10 & 12 & 12 & - \\
\hline P. parviflora & leaf & $\mathrm{CHCl}_{3}$ & 10 & - & 15 & 8 & 9 & - \\
\hline & & $\mathrm{CH}_{3} \mathrm{OH}$ & 9 & - & 9 & 9 & 9 & - \\
\hline & & $\mathrm{H}_{2} \mathrm{O}$ & - & - & - & - & - & - \\
\hline & vine & $\mathrm{CHCl}_{3}$ & 11 & - & 15 & 12 & - & - \\
\hline & & $\mathrm{CH}_{3} \mathrm{OH}$ & 15 & - & 11 & 11 & 12 & - \\
\hline & & $\mathrm{H}_{2} \mathrm{O}$ & 16 & - & - & 10 & - & - \\
\hline S brasiliensis & leaf & $\mathrm{CHCl}_{3}$ & 15 & 18 & 12 & 13 & 12 & - \\
\hline & & $\mathrm{CH}_{3} \mathrm{OH}$ & 10 & 14 & 13 & 14 & 11 & 10 \\
\hline & & $\mathrm{H}_{2} \mathrm{O}$ & 15 & - & 12 & 13 & 14 & - \\
\hline & branch & $\mathrm{CHCl}_{3}$ & 13 & 15 & 16 & 12 & 16 & 16 \\
\hline
\end{tabular}




\begin{tabular}{|c|c|c|c|c|c|c|c|c|}
\hline \multirow[b]{2}{*}{ Species } & \multirow[b]{2}{*}{ Part } & \multirow[b]{2}{*}{ Solvent } & \multicolumn{6}{|c|}{ Average inhibition zone diameter (mm) } \\
\hline & & & Ms & $\mathrm{Ec}$ & $\mathrm{Sa}$ & So & Ss & $\mathrm{Ca}$ \\
\hline \multirow{8}{*}{ S. daphnoides } & \multirow{5}{*}{ leaf } & $\mathrm{CH}_{3} \mathrm{OH}$ & - & 13 & 14 & 14 & 14 & 15 \\
\hline & & $\mathrm{H}_{2} \mathrm{O}^{3}$ & 24 & - & 11 & 15 & 12 & - \\
\hline & & $\mathrm{CHCl}_{3}$ & 15 & 13 & 9 & 10 & 9 & - \\
\hline & & $\mathrm{CH}_{3} \mathrm{OH}$ & 12 & - & 11 & 10 & 11 & - \\
\hline & & $\mathrm{H}_{2} \mathrm{O}$ & - & - & - & 8 & - & - \\
\hline & \multirow[t]{3}{*}{ branch } & $\mathrm{CHCl}_{3}$ & 14 & 13 & 16 & 18 & 15 & - \\
\hline & & $\mathrm{CH}_{3} \mathrm{OH}$ & 18 & 12 & 15 & 13 & 13 & 10 \\
\hline & & $\mathrm{H}_{2} \mathrm{O}$ & 10 & 12 & 12 & 8 & - & - \\
\hline \multirow[t]{6}{*}{ S. obovatum } & \multirow[t]{3}{*}{ leaf } & $\mathrm{CHCl}_{3}$ & 14 & 20 & 11 & 12 & 17 & - \\
\hline & & $\mathrm{CH}_{3} \mathrm{OH}$ & 14 & 15 & 11 & 12 & 13 & - \\
\hline & & $\mathrm{H}_{2} \mathrm{O}$ & 11 & 9 & 12 & 8 & 13 & - \\
\hline & \multirow[t]{3}{*}{ branch } & $\mathrm{CHCl}_{3}$ & 15 & 18 & 11 & 13 & 15 & 9 \\
\hline & & $\mathrm{CH}_{3} \mathrm{OH}$ & 15 & 20 & 12 & 13 & 12 & 9 \\
\hline & & $\mathrm{H}_{2} \mathrm{O}$ & - & 9 & 10 & 10 & 12 & 20 \\
\hline \multirow[t]{6}{*}{ T. spruceanum } & \multirow[t]{3}{*}{ leaf } & $\mathrm{CHCl}_{3}$ & 26 & 10 & 10 & 8 & 9 & - \\
\hline & & $\mathrm{CH}_{3} \mathrm{OH}$ & 25 & 15 & 14 & 13 & 14 & 19 \\
\hline & & $\mathrm{H}_{2} \mathrm{O}$ & 25 & 15 & 13 & 16 & 14 & 19 \\
\hline & \multirow[t]{3}{*}{ branch } & $\mathrm{CHCl}_{3}$ & 11 & 11 & 10 & 15 & 15 & 25 \\
\hline & & $\mathrm{CH}_{3} \mathrm{OH}$ & 27 & 20 & 11 & 16 & 20 & 30 \\
\hline & & $\mathrm{H}_{2} \mathrm{O}$ & 25 & 16 & 8 & 17 & 16 & 25 \\
\hline \multirow{2}{*}{\multicolumn{2}{|c|}{$\begin{array}{l}\text { Rifampicin } \\
\text { Nistatin }\end{array}$}} & $\mathrm{H}_{2}^{2} \mathrm{O}$ & 30 & 21 & 16 & 21 & 20 & nd \\
\hline & & $\mathrm{H}_{2}^{2} \mathrm{O}$ & nd & nd & nd & nd & nd & 35 \\
\hline
\end{tabular}

Ms: M. smegmatis; Ec: E. coli; Sa: S. aureus; So: S. oralis; Ss: S. sanguis; Ca: C. albicans; - : negative result; nd: not determined.

TABLE III

Minimal inhibitory concentrations (MIC) of extracts from 12 Ducke Forest Reserve plant species

\begin{tabular}{|c|c|c|c|c|c|c|c|c|}
\hline \multirow[b]{2}{*}{ Species } & \multirow[b]{2}{*}{ Part } & \multirow[b]{2}{*}{ Solvent } & \multicolumn{6}{|c|}{$\mathrm{MIC}(\mu \mathrm{g} / \mathrm{ml})$} \\
\hline & & & Ms & $\mathrm{Ec}$ & So & $\mathrm{Sa}$ & Ss & $\mathrm{Ca}$ \\
\hline C. kappleri & Branch & $\mathrm{CH}_{3} \mathrm{OH}$ & $>1000$ & nd & nd & nd & nd & nd \\
\hline \multirow[t]{4}{*}{ D. calycina } & Leaf & $\mathrm{CH}_{3} \mathrm{OH}$ & 195.0 & 195.0 & 97.7 & 48.8 & 195.0 & nd \\
\hline & Leaf & $\mathrm{CHCl}_{3}$ & 781.3 & 97.7 & 48.8 & 48.8 & 48.8 & nd \\
\hline & Branch & $\mathrm{CH}_{3} \mathrm{OH}$ & $>1000$ & nd & nd & nd & nd & nd \\
\hline & Branch & $\mathrm{CHCl}_{3}$ & 97.7 & 781.3 & nd & nd & 781.3 & nd \\
\hline D. johannesii & Branch & $\mathrm{H}_{2} \mathrm{O}^{3}$ & nd & $>1000$ & nd & nd & nd & nd \\
\hline \multirow[t]{2}{*}{ E. nuda } & Leaf & $\mathrm{CH}_{3} \mathrm{OH}$ & $>1000$ & nd & nd & nd & nd & $>1000$ \\
\hline & Leaf & $\mathrm{H}_{2} \mathrm{O}$ & $>1000$ & $>1000$ & nd & nd & nd & nd \\
\hline \multirow[t]{2}{*}{ L. gracilis } & Branch & $\mathrm{CHCl}_{3}$ & 48.8 & 781.3 & 390.6 & nd & nd & $>1000$ \\
\hline & Branch & $\mathrm{H}_{2} \mathrm{O}^{3}$ & nd & nd & nd & nd & nd & $>1000$ \\
\hline \multirow{2}{*}{ M. guianensis } & Leaf & $\mathrm{H}_{2}^{2} \mathrm{O}$ & nd & nd & nd & $>1000$ & nd & nd \\
\hline & Branch & $\mathrm{H}_{2}^{2} \mathrm{O}$ & nd & nd & nd & nd & nd & $>1000$ \\
\hline P. grandiflora & Branch & $\mathrm{CH}_{3}^{2} \mathrm{OH}$ & $>1000$ & nd & nd & nd & nd & nd \\
\hline P. parviflora & Vine & $\mathrm{CHCl}_{3}$ & - & nd & 781.3 & nd & nd & nd \\
\hline S. brasiliensis & Branch & $\mathrm{H}_{2} \mathrm{O}^{3}$ & $>1000$ & nd & nd & nd & nd & nd \\
\hline \multirow[t]{3}{*}{ S. obovatum } & Leaf & $\mathrm{CHCl}_{3}$ & nd & 781.3 & nd & nd & nd & nd \\
\hline & Branch & $\mathrm{CH}_{3} \mathrm{OH}$ & nd & $>1000$ & nd & nd & nd & nd \\
\hline & Branch & $\mathrm{H}_{2} \mathrm{O}$ & nd & nd & nd & nd & nd & $>1000$ \\
\hline \multirow[t]{6}{*}{ T. spruceanum } & Leaf & $\mathrm{CH}_{3} \mathrm{OH}$ & $>1000$ & nd & nd & nd & nd & nd \\
\hline & Leaf & $\mathrm{CHCl}_{3}$ & $>1000$ & nd & nd & nd & nd & nd \\
\hline & Leaf & $\mathrm{H}_{2} \mathrm{O}^{3}$ & 390.6 & nd & nd & nd & nd & nd \\
\hline & Branch & $\mathrm{CH}_{3}^{2} \mathrm{OH}$ & 781.3 & $>1000$ & nd & nd & $>1000$ & $>1000$ \\
\hline & Branch & $\mathrm{CHCl}_{3}$ & $>1000$ & nd & nd & nd & nd & $>1000$ \\
\hline & Branch & $\mathrm{H}_{2} \mathrm{O}^{3}$ & $>1000$ & nd & nd & nd & nd & $>1000$ \\
\hline
\end{tabular}

Ms: M. smegmatis; Ec: E. coli; Sa: S. aureus; So: S. oralis; Ss: S. sanguis; Ca: C. albicans; nd: not determined (see Table II for extracts with inhibition halo diameter $<20 \mathrm{~mm}$ at $50 \mathrm{~g} / 1$ ). 
TABLE IV

Results from bioautography applied to extracts from plants from the Ducke Forest Reserve and thin-layer chromatography (TLC) retention factors $\left(\mathrm{R}_{\mathrm{fs}}\right)$ of active antimicrobial spots

\begin{tabular}{|c|c|c|c|c|c|c|c|c|}
\hline \multirow[b]{3}{*}{ Species } & \multirow[b]{3}{*}{ Part } & \multirow[b]{3}{*}{ Solvent } & \multicolumn{6}{|c|}{$\mathrm{R}_{\mathrm{f}}$} \\
\hline & & & \multicolumn{5}{|c|}{ Antibacterial activity } & \multirow{2}{*}{$\frac{\text { Antifungal activity }}{\mathrm{Ca}}$} \\
\hline & & & Ms & $\mathrm{Ec}$ & So & $\mathrm{Sa}$ & Ss & \\
\hline \multirow[t]{9}{*}{ D. calycina } & Leaf & $\mathrm{CH}_{3} \mathrm{OH}$ & 0.93 & 0.71 & - & 0.78 & - & 0.81 \\
\hline & Leaf & $\mathrm{CHCl}_{3}$ & 0.93 & 0.70 & - & 0.55 & 0.5 & 0.78 \\
\hline & & & & & & 0.86 & 0.39 & \\
\hline & & & & & & & 0.28 & \\
\hline & Branch & $\mathrm{CH}_{3} \mathrm{OH}$ & 0.75 & - & - & - & - & - \\
\hline & Branch & $\mathrm{CHCl}_{3}$ & 0.47 & 0.55 & 0.56 & 0.86 & 0.59 & 0.8 \\
\hline & & & 0.36 & & 0.66 & 0.77 & 0.42 & \\
\hline & & & 0.24 & & & 0.52 & 0.27 & \\
\hline & & & 0.20 & & & 0.16 & & \\
\hline D. johannesii & Branch & $\mathrm{H}_{2} \mathrm{O}$ & nd & - & nd & nd & nd & nd \\
\hline E. nuda & Leaf & $\mathrm{H}_{2}^{2} \mathrm{O}$ & - & nd & 0.5 & nd & nd & nd \\
\hline \multirow[t]{5}{*}{ L. gracilis } & Leaf & $\mathrm{CHCl}_{3}$ & nd & - & - & - & - & - \\
\hline & Branch & $\mathrm{CHCl}_{3}^{3}$ & - & 0.46 & 0.49 & 0.32 & - & 0.39 \\
\hline & & & & 0.52 & 0.53 & & & \\
\hline & & & & & 0.23 & & & \\
\hline & Branch & $\mathrm{H}_{2} \mathrm{O}$ & - & - & - & - & - & - \\
\hline \multirow[t]{2}{*}{ M. guianensis } & Leaf & $\mathrm{H}_{2}^{2} \mathrm{O}$ & - & - & nd & nd & nd & nd \\
\hline & Branch & $\mathrm{H}_{2}^{2} \mathrm{O}$ & nd & - & nd & nd & nd & nd \\
\hline \multirow[t]{2}{*}{ P. grandiflora } & Branch & $\mathrm{CH}_{3} \mathrm{OH}$ & - & 0.83 & & & & \\
\hline & & & & 0.76 & nd & nd & nd & nd \\
\hline \multirow[t]{2}{*}{ P. parviflora } & Vine & $\mathrm{CHCl}_{3}$ & - & 0.83 & - & nd & nd & nd \\
\hline & & & & 0.77 & & & & \\
\hline S. obovatum & Leaf & $\mathrm{CHCl}_{3}$ & - & - & nd & - & nd & nd \\
\hline \multirow[t]{6}{*}{ T. spruceanum } & Leaf & $\mathrm{CH}_{3} \mathrm{OH}$ & - & - & nd & - & - & 0.40 \\
\hline & Leaf & $\mathrm{CHCl}_{3}$ & 0.27 & - & - & - & - & - \\
\hline & Leaf & $\mathrm{H}_{2} \mathrm{O}^{3}$ & - & - & nd & nd & - & - \\
\hline & Branch & $\mathrm{CH}_{3} \mathrm{OH}$ & - & - & 0.22 & nd & nd & - \\
\hline & Branch & $\mathrm{CHCl}_{3}$ & - & - & - & nd & nd & 0.16 \\
\hline & Branch & $\mathrm{H}_{2} \mathrm{O}$ & - & - & nd & - & nd & nd \\
\hline
\end{tabular}

Ms: M. smegmatis; Ec: E. coli; Sa: S. aureus; So: S. oralis; Ss: S. sanguis; Ca: C. albicans; -: negative result; nd: not determined.

M. smegmatis is an important test model for initial, primary screening for antimycobacterial activity, which is important in the search for drugs with potential antituberculosis effects. Mycobacterium tuberculosis (tuberculosis causing agent) is usually used at a later stage for further studies. M. smegmatis (ATCC 607) has been employed in bioassays and is cited many times, erroneously, in many publications as M. tuberculosis 607 . It is chosen as a model for tuberculosis since it does not present the pathogenic properties ascribed to $M$. tuberculosis and it exhibits rapid growth, in contrast to M. tuberculosis, with which slow growth is normally associated (Reyrat \& Kahn 2001, Newton et al. 2002, Okunade et al. 2004, Pauli et al. 2005).

Several screening studies have been performed to evaluate in vitro antimycobacterial activity of plant extracts. Tosun et al. (2004), using M. tuberculosis $\mathrm{H}_{37} \mathrm{Ra}$ as test microorganism, and an ethnobotanic approach for plant selection, evaluated extracts from 44 plant species belonging to 17 families and found that 23 extracts inhibited growth at concentrations from 50 to $200 \mu \mathrm{g} / \mathrm{ml}$. Billo et al. (2005) evaluated 22 plants (55 extracts) used in traditional medicine for treatment of symptoms potentially related to tuberculosis, representing 16 families, using $M$. bovis BCG as test organism, and found that five species exhibited activity towards this test organism.

Many plant species present inhibition zones of differing diameters, however, size difference of the inhibition zone depends primarily upon these factors: (a) diffusion capacity of substances (present in the extracts) in the agar medium, (b) antimicrobial activity of diffused substances, (c) origin of microorganisms, (d) $\mathrm{pH}$ of substrates in plates, (e) density of inoculation, and (f) growth and metabolic activity of microorganisms in the medium. This suggests that inhibitory activity is not necessarily proportional to the inhibition zone diameter, especially when comparing different extracts. Inhibition zone diameter can further be associated with polarities of substances which make up 
the tested extracts and also with cell wall composition of test organisms since Gram-positive bacteria present cell walls with lower lipid levels than do Gram-negative bacteria (Pauli et al. 2005, Bandeira et al. 2006).

Mycobacterium cell walls have elevated levels of high molecular weight lipids. This feature of cell wall structure seems to function as a barrier to the direct absorption of polar compounds (Pauli et al. 2005) and may be responsible for the more promising results obtained in the present study for chloroform extracts which have the lowest polarity among the extracts tested.

The present study made use of simple, rapid and inexpensive techniques for in vitro evaluation of antimicrobial activity. A similar method was used in recent studies to evaluate the activity of plant extracts and fractions in strains of $S$. aureus, E. coli and Pseudomonas aeruginosa (Chah et al. 2006), Streptococcus mitis, Streptococcus mutans and S. sanguis (Melo et al. 2006), as well as, $C$. albicans and other fungi which cause opportunistic infections (Lima et al. 2005). Alves et al. (2000), using the same method employed in the present study, namely diffusion in agar (wells were $7 \mathrm{~mm}$ in diameter), considered extracts with inhibition zones with diameters between 9 and 12 $\mathrm{mm}$ to be partially active, between 13 and $18 \mathrm{~mm}$ to be active, and greater than $18 \mathrm{~mm}$ to be very active. In the present study, extracts having inhibition zones with diameters greater than $20 \mathrm{~mm}$ were considered to be very active.

Bioautography revealed promising extracts from $D$. calycina. These results not only confirmed the activity of the leaf chloroform extract of this species detected in the other assays, but also revealed the antimicrobial potential of substances present in the branchwood chloroform extract, which were active in all microorganisms tested. Bioautography also revealed six extracts having components with inhibitory effects towards $C$. albicans. This activity towards $C$. albicans observed in bioautography contrasts strikingly with the results of MIC evaluation using this same species which revealed no significant activity for any of the extracts.

A considerable difference was shown to exist in the results obtained using diffusion in agar and bioautography. For example, L. gracillis branch chloroform extract, which presented the lowest MIC $(48.8 \mu \mathrm{g} / \mathrm{ml})$ using $M$. smegmatis in the diffusion method, was inactive in bioautography. On the other hand, D. calycina branch chloroform extract, which presented inhibition zone diameter $>20 \mathrm{~mm}$ and good inhibitory activity (MIC $<97.7 \mu \mathrm{g} / \mathrm{ml}$ ) only towards $M$. smegmatis, under bioautography conditions, presented active (growth inhibiting) substances (multiple Rfs) for all microorganisms evaluated.

Based on these results, D. calycina (Annonaceae) e $L$. gracilis (Apocynaceae) are promising antimicrobial plant species which need further study to reveal the identity of the antimicrobial substances present in their extracts. It should be stressed that the antimicrobial activity presented for these plant species is new information, having no basis in popular use or direct relation to previously published laboratory studies. These results contribute to the knowledge of bioactive species in the Amazon flora which, through further study, can reveal new lead compounds for drug development.

\section{REFERENCES}

Alves TMA, Ribeiro FL, Kloos H, Zani CL 2001. Polygodial, the fungitoxic component from the brazilian medicinal plant Polygonum puctatum. Mem Inst Oswaldo Cruz 96: 831-833.

Alves TMA, Silva AF, Brandão M, Grandi TSM, Smânia EFA, Smânia Jr A, Zani CL 2000. Biological screening of brazilian medicinal plants. Mem Inst Oswaldo Cruz 95: 367-373.

Bandeira MFCL, Teixeira MFS, Abinader CD, Parente RC, Lima PSL 2006. Avaliação in vitro da sensibilidade da Candida albicans ao hidróxido de cálcio associado ao óleo de copaíba. Rev Dentística 6: 12-22.

Billo M, Cabalion P, Waikedre J, Fourneau C, Bouttier S, Hocquemiller R, Fournet A 2005. Screening of some new Caledonian and Vanuatu medicinal plants for antimycobacterial activity. $J$ Ethnopharmacol 96: 195-200.

Botion LM, Ferreira AVM, Côrtes SF, Lemos VS, Braga FC 2005. Effects of the Brazilian phytopharmaceutical product Ierobina ${ }^{\circledR}$ on lipid metabolism and intestinal tonus. J Ethnopharmacol 102: 137-142.

Chah KF, Eze CA, Emuelosi CE, Esimone CO 2006. Antibacterial and wound healing properties of methanolic extracts of some nigerian medicinal plants. J Ethnopharmacol 104: 164-167.

Corrêa MP 1984. Dicionário das Plantas Úteis do Brasil e das Exóticas Cultivadas, Vol. 6, Ministério da Agricultura, Instituto Brasileiro de Desenvolvimento Florestal, Imprensa Nacional, Brasília, 777 pp.

Coykendall AL1989. Classification and identification of the viridans Streptococci. J Ethnopharmacol 2: 315-328.

Esmerino LA, Pereira AV, Schelesky ME 2005. Doseamento da potência da ciprofloxacina em comprimidos orais. Rev Bras Farm 86: $17-20$.

Fenner R, Betti AH, Mentz LA, Rates SMK 2006. Plantas utilizadas na medicina popular brasileira com potencial atividade antifúngica. Braz J Pharm Sci 42: 369-394.

Flora Project 2006. Available from: http://curupira.inpa.gov.br/projetos/ducke/ index.html [cited 2006 Dec 15].

Gayoso CW, Lima EO, Souza EL, Trajano VN, Pereira FOE, Lima IO 2004. Ação Inibitória do óleo essencial de Cinnamonum zeylanicum Blume, $\alpha$-pineno e $\beta$-pineno sobre fungos isolados de onicomicose. J Bras Fitomed 2: 25-29.

Gendron R, Grenier D, Maheu-Robert L 2000. The oral cavity as a reservoir of bacterial pathogens for focal infections. Microbes Infect 2: 897-906.

Giogetti M, Negri G, Rodrigues E 2007. Brazilian plants with possible action on the central nervous system - a study of historical sources from the 16th to 19th century. J Ethnopharmacol 109: 338-347.

Gurib-Fakim G 2006. Medicinal plants: traditions of yesterday and drugs of tomorrow. Mol Aspects Med 27: 1-93.

Hamada S, Slade H 1980. Biology, immunology, and cariogenicity of Streptococcus mutans. Microbiol Rev 44: 331-384.

Holetz FB, Pessini GL, Sanches NR, Cortez DAG, Nakamura CV, Dias Filho BP 2002. Screening of some plants used in the brazilian folk medicine for the treatment of infections diseases. Mem Inst Oswaldo Cruz 97: 1027-1031.

Koo H, Gomes BPFA, Rosalen PL, Ambrosano GMB, Park YK, Cury JA 2000. In vitro antimicrobial activity of propolis and Arnica montana against oral pathogens. Arch Oral Biol 45: 141-148.

Lima IO, Araújo R, Oliveira RAG, Lima EO, Souza EL, Farias NP, Navarro DF 2005. Inhibitory effect of some phytochemicals in 
the growth of yeasts potentially causing opportunistic infections. Braz J Pharm Sci 41: 199-203.

Melo AFM, Santos EJV, Souza LFC, Carvalho AAT, Pereira MSV, Higino JS 2006. Atividade antimicrobiana in vitro do extrato de Anacardium occidentale L. sobre espécies de Streptococcus. Rev Bras Farmacogn 16: 202-205.

Melo-Souza SE 2000. Tratamento das Doenças Neurológicas, Guanabara Koogan, Rio de Janeiro, 849 pp.

Montanari CA, Bolzani VS 2001. Planejamento racional de fármacos baseado em produtos naturais. Quím Nova 24: 105-111.

Moreira AC, Müller ACA, Pereira Jr N, Antunes MAS 2006. Pharmaceutical patents on plant derived materials in Brazil: policy, law and statistics. World Patent Inf 28: 34-42.

Motsei ML, Lindsey KL, Staden JE, Jäger AK 2003. Screening of traditionally used African plants for antifungal activity against Candida albicans. J Ethnopharmacol 86: 235-241.

Newton SM, Lau C, Gurcha SS, Besra GS, Wright CW 2002. The evaluation of forty-three plant species for in vitro antimycobacterial activities; isolation of active constituents from Psoralea corylifolia and Sanguinaria canadensis. J Ethnopharmacol 79: 57-67.

Okunade AL, Elvin-Lewis PF, Lewis WH 2004. Natural antimcobacterial metabolites: current status. Phytochemistry 65: 1017-1032.

Pauli GF, Case RJ, Inui T, Wang Y, Cho S, Fischer NH, Franzblau SG 2005. New perspectives on natural products in TB drug research. Life Sci 78: 485-494.

Pereira JV, Bergamo DCB, Pereira JO, França SC, Pietro RCLR, Souza-Silva YTC 2005. Antimicrobial activity of Arctium lappa constituents against microorganisms commonly founding endodontic infections. Braz Dent J 16: 192-196.

Piovano S 1999. Bacteriology of most frequent oral anaerobic infections. Anaerobe 5: 221-227.

Pohlit AM, Quignard ELJ, Nunomura SM, Tadei WP, Hidalgo AF, Pinto ACS, Santos EVM, Morais SKR, Saraiva RCG, Ming LC,
Alecrim AM, Ferraz AB, Pedroso ACS, Diniz EV, Finney EK, Gomes EO, Dias HB, Souza KS, Oliveira LCP, Don LC, Queiroz MMA, Henrique MC, Santos M, Lacerda Júnior OS, Pinto PS, Silva SG, Graça YR 2004. Screening of plants found in the State of Amazonas, Brazil for larvicial activity against Aedes aegypti larvae. Acta Amazônica 34: 97-105.

Quignard ELJ, Nunomura SM, Pohlit AM, Alecrim AM, Pinto ACS, Portela CN, Oliveira LCP, Don LC, Silva LFR, Henrique MC, Santos M, Pinto PS, Silva SG 2004. Median Lethal Concentrations of Amazonian Plant Extracts in the Brine Shrimp Assay. Pharm Biol 42: 253-257.

Quignard ELJ, Pohlit AM, Nunomura SM, Pinto ACS, Santos EVM, Morais SKR, Alecrim AM, Pedroso ACS, Cyrino BRB, Melo CS, Finney EK, Gomes EO, Souza KS, Oliveira LCP, Don LC, Silva LFR, Queiroz MMA, Henrique MC, Santos M, Pinto OS, Silva SG 2003. Screening plants found Amazonas state for lethality towards brine shrimp Artemia franciscana. Acta Amazônica 33: 93-104.

Reyrat JM, Kahn D 2001. Mycobacterium smegmatis: an absurd model for tuberculosis? Trends Microbiol 9: 472-473.

Ribeiro JELS, Hopkins MJG, Vicentini A, Sothers CA, Costa MAS, Brito JM, Souza MAD, Martins LHP, Lohmann LG, Assunção PACL, Pereira EC, Silva CF, Mesquita MR, Procópio LC 1999. Flora da Reserva Ducke: guia de identificação das plantas vasculares de uma floresta de terra-firme na Amazônia Central, INPA, Manaus, $816 \mathrm{pp}$.

Silva MF, Lisbôa PLB, Lisbôa RCL 1977. Nomes Vulgares de Plantas Amazônicas, INPA, Belém, 222 pp.

Tosun F, Kizilay ÇA, Sener B, Vural M, Palittapongarnpim P 2004. Antimycobacterial screening of some Turkish plants. $J$ Ethnopharmacol 95: 273-275.

Turolla MSR, Nascimento ES 2006. Informações toxicológicas de alguns fitofármacos utilizados no Brasil. Rev Bras Cien Farm 42: 289-306.

Wade WG, Munson MA, Lillo A, Weightman AJ 2005. Specificity of the oral microflora in dentinal caries, endodontic infections and periodontitis. Int Congr Ser 1284: 150-157. 